\title{
Mass and angular momentum of asymptotically AdS or flat solutions in the topologically massive gravity
}

\author{
Serkay Ölmez * Özgür Sarığlu $丹$ and Bayram Tekin \\ Department of Physics, Faculty of Arts and Sciences, \\ Middle East Technical University, 06531, Ankara, Turkey
}

(Dated: June 25, 2018)

\begin{abstract}
We study the conserved charges of supersymmetric solutions in the topologically massive gravity theory for both asymptotically flat and constant curvature geometries.

PACS numbers: 04.60.Kz, 11.10.Kk, 04.30.-w, 04.90.+e
\end{abstract}

\section{INTRODUCTION}

In the presence of a cosmological constant, the source-free field equations of the $(2+1$ dimensional) topologically massive gravity (TMG) theory read

$$
R_{\mu \nu}-\frac{1}{2} g_{\mu \nu} R+\Lambda g_{\mu \nu}+\frac{1}{\mu} C_{\mu \nu}=0, \quad C^{\mu \nu} \equiv \frac{1}{\sqrt{-g}} \epsilon^{\mu \alpha \beta} \nabla_{\alpha}\left(R_{\beta}^{\nu}-\frac{1}{4} \delta_{\beta}^{\nu} R\right)
$$

where the Cotton tensor $C_{\mu \nu}$ is the three dimensional analogue of the Weyl tensor and is symmetric, traceless and identically conserved; the parameter $\mu$ is the coupling constant for the gravitational Chern-Simons term in the action and corresponds to the mass of the linearized TMG excitations at $\Lambda=0$ (see [1] for details and for $\Lambda \neq 0$ see [2]). A minimally supersymmetric extension of this theory was also constructed long time ago [3].

The well known BTZ metric [4], as well as the Anti-de Sitter (AdS) and the SchwarzschilddS spacetimes, are solutions to TMG theory with a cosmological constant in a 'trivial' manner since their Cotton tensors vanish identically. There are, however, other known 'nontrivial' solutions; i.e. those spacetimes that obey the 'full' TMG equations, not just their Einstein part (or the cosmological Einstein part in the relevant cases) alone. The first example that we know is Deser's gravitational anyons which are only solutions of the linearized TMG equations [5]. The 'fully' nonlinear, 'nontrivial' solutions include the Vuorio solution [6] and its generalization to solutions with a constant twist [7]. There are also exact static/stationary solutions for spinning point sources for which the spin and the mass of the sources obey a certain relation [8], 9]. A class of cosmological-type solutions is given by finite action exact solutions of TMG [10] that are also useful for a classification of homogeneous solutions. There also exists a two parameter solution to TMG theory with a cosmological constant which seems to have properties similar to the BTZ solution [11], [12]. This solution is not asymptotically AdS in its original form, but then its asymptotically AdS form, which is obtained by imposing a certain relation between $\Lambda$ and $\mu$, is equivalent to the BTZ metric.

*Electronic address: olmezs@newton.physics.metu.edu.tr

$\dagger$ Electronic address: sarioglu@metu.edu.tr

${ }^{\ddagger}$ Electronic address: btekin@metu.edu.tr 
Another class of solutions which asymptotically approach extremal BTZ black holes, but are geodesically complete with no event horizons, were also given [13]. Finally, the first nontrivial example of a solution to TMG that preserves half of supersymmetry was found in 14]; moreover, the solutions in [13] seem to be related to the supersymmetric solutions by a certain choice of parameters and a coordinate transformation. Another two-parameter family of black hole solutions that are obtained by an analytical continuation of the Vuorio solution, but fail to be asymptotically AdS like their ancestor, was recently given in [15].

In a recent work [16], a concrete and rigorous definition of conserved gravitational charges (particularly energy and angular momentum) were given in a 'surface' integral form about their flat or asymptotically AdS backgrounds in TMG theory. It is only natural to consider the exact solutions listed in the previous paragraph as explicit examples whose gravitational charges can be calculated a la [16]. We do this for the BTZ and the only nontrivial supersymmetric solutions of TMG that are asymptotically AdS or flat in this paper. This should also help to better understand the physical properties of these examples and to clarify the physical meanings of some of the parameters that explicitly show up in them.

\section{THE CONSERVED GRAVITATIONAL CHARGES OF THE TMG THEORY}

Let us start by giving a brief outline of how gravitational charges are defined in TMG. (We refer the reader to [16] and [17] for details.) Assume that the deviation, $h_{\mu \nu}$, of the actual spacetime metric $g_{\mu \nu}=\bar{g}_{\mu \nu}+h_{\mu \nu}$ from an asymptotically AdS metric (or the background) $\bar{g}_{\mu \nu}$, which obeys

$$
\bar{R}_{\mu \alpha \nu \beta}=\Lambda\left(\bar{g}_{\mu \nu} \bar{g}_{\alpha \beta}-\bar{g}_{\mu \beta} \bar{g}_{\alpha \nu}\right), \quad \bar{R}_{\mu \nu}=2 \Lambda \bar{g}_{\mu \nu}, \quad \bar{R}=6 \Lambda,
$$

is employed for constructing "linearized gravity" in the usual sense with the usual assumptions [17]. Then the 'linearized' part of the Ricci tensor [29]

$$
R_{\mu \nu}^{L}=\frac{1}{2}\left(-\bar{\square} h_{\mu \nu}-\bar{\nabla}_{\mu} \bar{\nabla}_{\nu} h+\bar{\nabla}^{\sigma} \bar{\nabla}_{\nu} h_{\sigma \mu}+\bar{\nabla}^{\sigma} \bar{\nabla}_{\mu} h_{\sigma \nu}\right),
$$

and the linearized Ricci scalar

$$
R^{L} \equiv\left(R_{\mu \nu} g^{\mu \nu}\right)^{L}=R_{\mu \nu}^{L} \bar{g}^{\mu \nu}-2 \Lambda h=-\bar{\square} h+\bar{\nabla}_{\mu} \bar{\nabla}_{\nu} \bar{h}^{\mu \nu}-2 \Lambda h,
$$

can be used for finding the linearized cosmological Einstein and the Cotton tensors as

$$
\begin{aligned}
\mathcal{G}_{\mu \nu} & \equiv\left(G_{\mu \nu}+\Lambda g_{\mu \nu}\right)^{L}=R_{\mu \nu}^{L}-\frac{1}{2} \bar{g}_{\mu \nu} R^{L}-2 \Lambda h_{\mu \nu}, \\
C_{L}^{\mu \nu} & =\frac{1}{\sqrt{-\bar{g}}} \epsilon^{\mu \alpha \beta} \bar{g}_{\beta \sigma} \bar{\nabla}_{\alpha}\left(R_{L}^{\sigma \nu}-2 \Lambda h^{\sigma \nu}-\frac{1}{4} \bar{g}^{\sigma \nu} R_{L}\right) .
\end{aligned}
$$

Now one can find a background conserved and gauge invariant charge (corresponding to each background Killing vector $\bar{\xi}^{\mu}$ ) which is given as the sum of the following three terms:

$$
Q^{\mu}(\bar{\xi})=\frac{1}{8 \pi G} \oint_{\partial \mathcal{M}} d S_{i}\left(q_{E}^{\mu i}(\bar{\xi})+\frac{1}{2 \mu} q_{E}^{\mu i}(\bar{\Xi})+\frac{1}{2 \mu} q_{C}^{\mu i}(\bar{\xi})\right),
$$


where [30]

$$
\begin{aligned}
q_{E}^{\mu i}(\bar{\xi}) \equiv \sqrt{-\bar{g}}\left(\bar{\xi}_{\nu} \bar{\nabla}^{\mu} h^{i \nu}-\bar{\xi}_{\nu} \bar{\nabla}^{i} h^{\mu \nu}+\bar{\xi}^{\mu} \bar{\nabla}^{i} h-\bar{\xi}^{i} \bar{\nabla}^{\mu} h\right. \\
\left.\quad+h^{\mu \nu} \bar{\nabla}^{i} \bar{\xi}_{\nu}-h^{i \nu} \bar{\nabla}^{\mu} \bar{\xi}_{\nu}+\bar{\xi}^{i} \bar{\nabla}_{\nu} h^{\mu \nu}-\bar{\xi}^{\mu} \bar{\nabla}_{\nu} h^{i \nu}+h \bar{\nabla}^{\mu} \bar{\xi}^{i}\right), \\
q_{C}^{\mu i}(\bar{\xi}) \equiv \epsilon^{\mu i \beta} \mathcal{G}_{\nu \beta} \bar{\xi}^{\nu}+\epsilon^{\nu i \beta} \mathcal{G}^{\mu}{ }_{\beta} \bar{\xi}_{\nu}+\epsilon^{\mu \nu \beta} \mathcal{G}^{i}{ }_{\beta} \bar{\xi}_{\nu},
\end{aligned}
$$

and $\bar{\Xi}^{\beta} \equiv \epsilon^{\alpha \nu \beta} \bar{\nabla}_{\alpha} \bar{\xi}_{\nu} / \sqrt{-\bar{g}}$ is another background Killing vector constructed out of $\bar{\xi}$. Here $\mathcal{M}$ is a spatial 2-dimensional hypersurface, $\partial \mathcal{M}$ is its 1-dimensional boundary and $i$ denotes the space direction orthogonal to the boundary $\partial \mathcal{M}$ with the corresponding line element $d S_{i}$. $G$ denotes the 3-dimensional Newton's constant and the charge has been normalized by the overall factor $8 \pi G$ in (11).

\section{THE BTZ BLACK HOLE}

To set the stage properly, let us take the BTZ solution [4]

$$
d s^{2}=\left(M-\frac{r^{2}}{\ell^{2}}\right) d t^{2}-J d t d \phi+r^{2} d \phi^{2}+\frac{d r^{2}}{-M+\frac{r^{2}}{\ell^{2}}+\frac{J^{2}}{4 r^{2}}}
$$

as a first example. The correct black hole vacuum background is found by setting $M=0$, $J=0$ in (4) (see [4] for a discussion on this) which is clearly locally AdS:

$$
d s^{2}=-\frac{r^{2}}{\ell^{2}} d t^{2}+\frac{\ell^{2}}{r^{2}} d r^{2}+r^{2} d \phi^{2} .
$$

The timelike $\bar{\xi}^{\mu}=(-\partial / \partial t)^{\mu}$ and the spacelike $\bar{\zeta}^{\mu}=(\partial / \partial \phi)^{\mu}$ Killing vectors can be used in finding the conserved energy and the angular momentum, respectively. The surface integral (11) at some finite distance $r$ from the origin yields the following non gauge-invariant quantities, which afterwards give the 'true' energy and angular momentum that are only to be measured at infinity [31]:

$$
\begin{aligned}
& E(r)=\frac{4 r^{4}\left(J-\mu M \ell^{2}\right)+J \ell^{2} r^{2}(\mu J-4 M)+J^{3} \ell^{2}}{-4 \mu \ell^{2} r^{4}+4 \mu M \ell^{4} r^{2}-\mu J^{2} \ell^{4}} \\
& L(r)=\frac{8 r^{5}(\mu J-M)+J r^{3}\left(J-8 \mu M \ell^{2}\right)+J^{2} \ell^{2} r(2 \mu J-M)}{2 \mu r\left(4 r^{4}-4 M \ell^{2} r^{2}+J^{2} \ell^{2}\right)} .
\end{aligned}
$$

As a result, one obtains the energy and the angular momentum in the limit as $r \rightarrow \infty$ to be

$$
E=M-\frac{J}{\mu \ell^{2}} \quad \text { and } \quad L=J-\frac{M}{\mu} .
$$

These quantities [15, 18] are obviously different from the ADM charges of the BTZ black hole [4]; the Cotton part clearly has a nontrivial contribution to the conserved charges. Amusingly enough, the angular momentum vanishes when the two parameters $M$ and $J$ are related by $M=\mu J$, in which case $E=M\left(1-1 /\left(\mu^{2} \ell^{2}\right)\right)$. Thus, if furthermore $\mu^{2} \ell^{2}=1$, then the BTZ black hole is left with no 'energy' and 'angular momentum' in the TMG context!

As a brief remark on the charged version of the BTZ solution [19], we note that since the "electric potential" rises logarithmically in $D=3$, even a cursory look suggests that a single charged black hole will have divergent energy. In fact the authors of [19] define the energy of their charged rotating solution only upto an infinite constant factor. In this respect, the gauge invariant energy in the sense of [16] is naturally found to be divergent for this case. 


\section{THE SUPERSYMMETRIC SOLUTION}

The half supersymmetry preserving solution given in [14] is described by the metric

$$
d s^{2}=-f^{2}(\rho) d t^{2}+d \rho^{2}+h^{2}(\rho)[d \phi+a(\rho) d t]^{2}
$$

and depending on whether the cosmological constant $\Lambda=-1 / \ell^{2}<0$ is present or not, the metric functions are given by either 32]

i) nonvanishing cosmological constant:

$$
\begin{aligned}
& f(\rho)=f_{0} e^{2 \rho / \ell} X^{-1 / 2}, \quad h(\rho)=h_{0} X^{1 / 2}, \quad a(\rho)=-a_{0}+k \frac{f_{0}}{h_{0}} e^{2 \rho / \ell} X^{-1}, \\
& X(\rho) \equiv \beta_{0}+\beta_{1} e^{2 \rho / \ell}+\beta_{2} e^{(1 / \ell-\mu k) \rho}
\end{aligned}
$$

or

ii) vanishing cosmological constant:

$$
\begin{aligned}
& f(\rho)=f_{0} Y^{-1 / 2}, \quad h(\rho)=h_{0} Y^{1 / 2}, \quad a(\rho)=-a_{0}+k \frac{f_{0}}{h_{0}} Y^{-1} \\
& Y(\rho) \equiv \beta_{3} e^{-\mu k \rho}-\mu \beta_{4}\left(\omega_{0}+k \rho\right) .
\end{aligned}
$$

Here $f_{0}, h_{0}, a_{0}, \beta_{i}(i=0,1, \ldots, 4)$ and $\omega_{0}$ are all real constants that arise from the integration of the field equations whereas $k= \pm 1$ is a free parameter that comes from the solution of the Killing spinor equation on the supersymmetry side.

In 14], it was impossible to explicitly invert the functional relation $r=h(\rho)$ for the case of the nonvanishing cosmological constant so that the metric could be brought to the wellstudied BTZ form [4], and the vast literature on that metric could be suitably adopted for an analysis of the physical meanings of the integration constants above. Instead, a much more complicated analysis was carried out by studying the quasilocal mass and the quasilocal angular momentum which was developed in [20] in an AdS background. We refer the reader to [14] for the details of this.

Here a brief remark stating the differences between these quasilocal charges and the gravitational charges in the sense of [16, 17] are in order perhaps: The quasilocal energy in a spatially bounded region (such as an asymptotically AdS background for our case) is defined as minus the 'time' rate of change of the classical gravitational action. An analogous definition exists also for the quasilocal angular momentum. (Please see 21] and the references therein for the attempts to define "quasilocal gravitational charges".) The definition of gravitational charges in TMG, however, are much more natural since these gauge invariant conserved (global) charges are forged into being by the Gauss law and the presence of asymptotic Killing symmetries [16, 17].

For the time being, let us concentrate on the case of nonvanishing cosmological constant $\Lambda=-1 / \ell^{2} \neq 0$. By substituting the metric functions (66) in the metric (15), one obtains

$$
d s^{2}=d \rho^{2}-\frac{f_{0}^{2} e^{4 \rho / \ell}}{X(\rho)} d t^{2}+\frac{f_{0}^{2} e^{4 \rho / \ell}}{X(\rho)}\left(d t+k \frac{h_{0}}{f_{0}} e^{-2 \rho / \ell} X(\rho)\left(d \phi-a_{0} d t\right)\right)^{2}
$$

after some simplifications.

In [14], it was found that the quasilocal mass was $a_{0}$ times the quasilocal angular momentum (see (39) of [14]) and the asymptotic behavior of the metric was examined through 
the metric function $a(r)$ and hence $a_{0}$. It was shown that for $a$ to vanish asymptotically as $r \rightarrow \infty, a_{0}$ had to be chosen either as 0 or as $k f_{0} /\left(h_{0} \beta_{1}\right)$. Whether $a_{0}$ has a physical meaning or not (and whether it can be set equal to zero or not), one should be able to make the simple change of variable $d \theta=d \phi-a_{0} d t$ in the metric (8). The outcome is simply

$$
d s^{2}=d \rho^{2}+2 k f_{0} h_{0} e^{2 \rho / \ell} d t d \theta+h_{0}^{2} X(\rho) d \theta^{2} .
$$

Another simple redefinition of the coordinates as $u=k f_{0} t$ and $v=h_{0} \theta$ can always be made at this stage and one arrives at the final form

$$
d s^{2}=d \rho^{2}+2 e^{2 \rho / \ell} d u d v+\left(\beta_{0}+\beta_{1} e^{2 \rho / \ell}+\beta_{2} e^{(1 / \ell-\mu k) \rho}\right) d v^{2} .
$$

It is obvious that one of the integration constants in (9) can be set to 1 by simple coordinate rescalings. The curvature invariants of this metric can be calculated easily: the Ricci scalar $R=-6 / \ell^{2}$ and $R_{\mu \nu} R^{\mu \nu}=12 / \ell^{4}$, moreover this solution is asymptotically AdS (for $1 / \ell-$ $\mu k<0$ ) with no curvature singularities. When $\beta_{0}=\beta_{2}=0$, the metric is the AdS metric in the Poincaré coordinates. For this case, even when one starts with $\beta_{1}=0$, one can still introduce it back by a simple coordinate redefinition as $\tilde{u}=u-\beta_{1} v / 2$.

There is yet another alternative way to understand the emergence of the constants $\beta_{0}$ and $\beta_{1}$ in the expression for $X(\rho)$. These two terms can be thought of as describing a gravitational wave in AdS. One can use the technique developed by Garfinkle and Vachaspati 22] which permits the addition of a wave to an already existing solution when there is a null Killing vector present. A detailed discussion of this method, its extension to various supergravity theories and to theories that include nontrivial matter couplings can be found in [23]. One can easily follow the footsteps of 23] and conclude without any difficulties that the Garfinkle-Vachaspati method is also applicable to the TMG theory. A brief outline of this technique can also be found in the appendix of [24] and here we will use the notation outlined there. In our case, one starts from (9) with $X(\rho)=0$, i.e. the AdS metric

$$
d s^{2}=d \rho^{2}+2 e^{2 \rho / \ell} d u d v
$$

which satisfies $R_{\mu \nu}=\left(-2 / \ell^{2}\right) g_{\mu \nu}$. Using the null Killing vector $k^{\mu}=(\partial / \partial v)^{\mu}$, the scalar $\Omega$ (of [24]) is calculated easily as $\Omega=\Omega_{0} e^{-2 \rho / \ell}$, where $\Omega_{0}$ is an arbitrary constant. Following relevant steps, one obtains $\Phi(\rho)=\Phi_{1}-\ell \Phi_{0} e^{-2 \rho / \ell}$, where $\Phi_{0}$ and $\Phi_{1}$ are arbitrary real constants. Using these, defining $-\Omega_{0} \ell \Phi_{0} \equiv \beta_{0}$ and $\Omega_{0} \Phi_{1} \equiv \beta_{1}$, one obtains (9) with $\beta_{2}$ set equal to zero in $X(\rho)$.

In fact the metric with $\beta_{2}=0$ has showed up earlier in different contexts: It corresponds to a generalized Kaigorodov metric [25]. It is obtainable from the AdS metric by an $S L(2, R)$ transformation [26] and its equivalence to the extremal limit of the BTZ black hole [4] can be shown [26, 27]. A crucial point is that the boundaries of the AdS and the extremal BTZ metric are different (see 27] for details). When $\beta_{2} \neq 0$, one can again remove the constant $\beta_{0}$ by a shift in the $\rho$ coordinate.

Another observation that needs to be stated is that in fact the constants $\beta_{0}, \beta_{1}$ and $\beta_{2}$ can be taken as arbitrary functions of $v$ and the metric (9) is also a solution to the TMG equations with a cosmological constant when $X(\rho)$ is replaced by

$$
X(\rho, v) \equiv \beta_{0}(v)+\beta_{1}(v) e^{2 \rho / \ell}+\beta_{2}(v) e^{(1 / \ell-\mu k) \rho} .
$$

These arbitrary functions can be thought of as describing the profile of the gravitational wave then. However when they are left arbitrary, it is highly probable that the supersymmetry is completely broken. 
Let us now calculate the gravitational charges associated with the metric (9) using the procedure outlined in section III. It is clear that the metric (10) can be used as the background with its timelike $\bar{\xi}^{\mu}=(-\partial / \partial u+\partial / \partial v)^{\mu}$ and spacelike $\bar{\zeta}^{\mu}=(\partial / \partial u+\partial / \partial v)^{\mu}$ Killing vectors yielding the energy and the angular momentum, respectively. After a tedious calculation, one finds that the integrand (that is, the terms inside the parentheses) in (II) is given by

$$
E(\rho)=\delta^{\mu}{ }_{u} \delta_{\rho}^{i} \frac{1}{2 \mu \ell^{2}}\left\{4 \beta_{0}(1+\mu \ell)+\beta_{2} e^{(1 / \ell-\mu k) \rho}(1+\mu k \ell)[1+(k+2) \mu \ell]\right\},
$$

and obviously depending on the sign of $1 / \ell-\mu k$, one finds that the energy at the boundary of $\operatorname{AdS}(\rho \rightarrow \infty)$ is $[33$ ]

$$
E=\left\{\begin{array}{cc}
2(k / \ell)(1+k)\left(\beta_{0}+\beta_{2}\right), & 1 / \ell-\mu k=0 \\
2 \beta_{0}(1+\mu \ell) /\left(\mu \ell^{2}\right), & 1 / \ell-\mu k<0
\end{array} .\right.
$$

As for the angular momentum, one finds that it is equal to the energy: $L=E$.

The steps that have been taken up until this point can also be repeated in an analogous fashion for the case of vanishing cosmological constant. The metric that corresponds to (8) in such a process is simply found as

$$
d s^{2}=d \rho^{2}+2 d t d \theta+\left(\beta_{3} e^{-\mu k \rho}-\mu \beta_{4}\left(\omega_{0}+k \rho\right)\right) d \theta^{2} .
$$

If one starts from the flat metric $d s^{2}=d \rho^{2}+2 d t d \theta$ and applies the Garfinkle-Vachaspati method to add a gravitational wave to this spacetime, one readily finds $\Omega=\Omega_{0}$ and $\Phi(\rho)=$ $\Phi_{1}+\Phi_{0} \rho$, where $\Phi_{0}, \Phi_{1}$ and $\Omega_{0}$ are arbitrary real constants, and these, with the definitions $\Omega_{0} \Phi_{1} \equiv-\mu \beta_{4} \omega_{0}$ and $\Omega_{0} \Phi_{0} \equiv-\mu k \beta_{4}$, lead to the metric (111) with $\beta_{3}$ set equal to zero in $Y(\rho)$.

Once again the constants $\beta_{4}, \omega_{0}$ and $\beta_{3}$ can be taken as arbitrary functions of $\theta$ and the metric (11) is also a solution to the TMG equations when $Y(\rho)$ is replaced by

$$
Y(\rho, \theta) \equiv \beta_{3}(\theta) e^{-\mu k \rho}-\mu \beta_{4}(\theta)\left(\omega_{0}(\theta)+k \rho\right) .
$$

These arbitrary functions can be thought of as describing the wave profile again, but with these functions in place, it may be that there is no supersymmetry left to preserve then.

As for the gravitational charges related to (11), the background to work with is simply the flat metric $d s^{2}=d \rho^{2}+2 d t d \theta$ and the Killing vectors needed are just the timelike $\bar{\xi}^{\mu}=(-\partial / \partial t+\partial / \partial \theta)^{\mu}$ and the spacelike $\bar{\zeta}^{\mu}=(\partial / \partial t+\partial / \partial \theta)^{\mu}$ vectors. The steps leading to (11) can easily be repeated by setting $\Lambda=0$ in the relevant places and replacing the covariant derivatives with respect to $\bar{g}_{\mu \nu}$ with ordinary derivatives. One then finds that the terms inside the parentheses in (11) is given by

$$
E(\rho)=\delta_{t}^{\mu} \delta_{\rho}^{i}\left(\mu k \beta_{4}+\frac{1}{2} \mu \beta_{3}(2 k-1) e^{-\mu k \rho}\right)
$$

and depending on the sign of $\mu k$, one finds the energy as $\rho \rightarrow \infty$ to be

$$
E=\left\{\begin{array}{cc}
0, & \mu=0 \\
\mu k \beta_{4}, & \mu k>0
\end{array}\right.
$$

The angular momentum is again given by $L=E$. 


\section{CONCLUSIONS}

In this paper, we showed how the physical properties of a known supersymmetric solution of the full cosmological TMG theory can be better understood by the Garfinkle-Vachaspati method and then determined its conserved charges for bulk asymptotically flat and constant curvature backgrounds. Even though the question of how the supersymmetric version of the TMG theory can be obtained from any compactification of M-theory or, for that matter, any higher dimensional supergravity theory remains open, provided that an exact form of the CFT dual of TMG can be formulated on the boundary of AdS, this particular supersymmetric solution should be suitable for understanding the AdS/CFT duality in the infinite momentum frame [26], 27]. Another open question that deserves attention is the problem of finding a supersymmetric matter coupled extension of the TMG theory. Looking for the charged versions of the metrics studied here within this model and their relations with the ones presented in [28] would be certainly worth the effort.

\section{ACKNOWLEDGMENTS}

We thank G. Clément for useful discussions. This work is partially supported by the Scientific and Technical Research Council of Turkey (TÜBİTAK); the work of B.T. is also supported by the "Young Investigator Fellowship" of the Turkish Academy of Sciences (TÜBA) and by a TÜBITAK Kariyer Grant.

[1] S. Deser, R. Jackiw and S. Templeton, Phys. Rev. Lett. 48975 (1982); Ann. Phys. (N.Y.) 140372 (1982); 185 406(E) (1988).

[2] S. Deser and B. Tekin, Class. Quantum Grav. 19 L97 (2002).

[3] S. Deser and J.H. Kay, Phys. Lett. 120B 97 (1983); S. Deser, in Quantum Theory of Gravity: Essays in Honor of the 60th Birthday of Bryce S. DeWitt, edited by S.M. Christensen (Adam Hilger, London, 1984), pp. 374-381.

[4] M. Bañados, C. Teitelboim and J. Zanelli, Phys. Rev. Lett. 691849 (1992); M. Bañados, M. Henneaux, C. Teitelboim and J. Zanelli, Phys. Rev. D 481506 (1993).

[5] S. Deser, Phys. Rev. Lett. 64611 (1990).

[6] I. Vuorio, Phys. Lett. 163B 91 (1985).

[7] R. Percacci, P. Sodano and I. Vuorio, Ann. Phys. (N.Y.) 176344 (1987).

[8] M.E. Ortiz, Class. Quantum Grav. 7 L9 (1990).

[9] A. Edery and M.B. Paranjape, Phys. Lett. 413B 35 (1997).

[10] Y. Nutku and P. Baekler, Ann. Phys. (N.Y.) 19516 (1989).

[11] Y. Nutku, Class. Quantum Grav. 102657 (1993).

[12] M. Gürses, Class. Quantum Grav. 112585 (1994).

[13] G. Clément, Class. Quantum Grav. 11 L115 (1994).

[14] T. Dereli and Ö. Sarığlu, Phys. Rev. D 64027501 (2001).

[15] K. Ait Moussa, G. Clément and C. Leygnac, Class. Quantum Grav. 20 L277 (2003).

[16] S. Deser and B. Tekin, Class. Quantum Grav. 20 L259 (2003).

[17] S. Deser and B. Tekin, Phys. Rev. Lett. 89101101 (2002); Phys. Rev. D 67084009 (2003).

[18] S. Deser, İ. Kanık and B. Tekin, Class. Quantum Grav. 223383 (2005). 
[19] C. Martínez, C. Teitelboim and J. Zanelli, Phys. Rev. D 61104013 (2000).

[20] T. Dereli and Yu. N. Obukhov, Phys. Rev. D 62024013 (2000).

[21] J.D. Brown and J.W. York, Jr, Phys. Rev. D 471407 (1993).

[22] D. Garfinkle and T. Vachaspati, Phys. Rev. D 421960 (1990); D. Garfinkle, Phys. Rev. D 46 4286 (1992).

[23] N. Kaloper, R.C. Myers and H. Roussel, Phys. Rev. D 557625 (1997).

[24] N.S. Değer and Ö. Sarı̆̆lu, J. High Energy Phys. 12039 (2004).

[25] V.R. Kaigorodov, Dokl. Akad. Nauk. SSSR 146793 (1962) [Sov. Phys. Doklady 7893 (1963)].

[26] D. Brecher, A. Chamblin and H.S. Reall, Nucl. Phys. B 607155 (2001).

[27] M. Cvetič, H. Lu and C.N. Pope, Nucl. Phys. B 545309 (1999).

[28] T. Dereli and Ö. Sarığlu, Phys. Lett. 492B 339 (2000).

[29] Here $h \equiv h_{\mu \nu} \bar{g}^{\mu \nu}$, all indices are raised and lowered with the background metric $\bar{g}_{\mu \nu}$ and also all covariant differentiations are carried with respect to $\bar{g}_{\mu \nu}$.

[30] Here one in fact has $\mathcal{G}^{\mu \nu} \equiv\left(G^{\mu \nu}+\Lambda g^{\mu \nu}\right)^{L}$ but then since $\bar{G}_{\mu \nu}+\Lambda \bar{g}_{\mu \nu}=0$, moving the indices of the linearized cosmological Einstein tensor $\mathcal{G}$ can equivalently be carried out with the background metric $\bar{g}_{\mu \nu}$.

[31] Throughout we have chosen the Newton constant $G$ in (11) such that one finds the usual ADM pair $(M, J)$ in the limit $\mu \rightarrow \infty$.

[32] The constants $\beta_{0}$ and $\beta_{3}$ were set equal to 1 in [14]. Here we keep them for later convenience.

[33] Here we have chosen the Newton constant $G$ in (11) accordingly. 